\title{
Chorioretinal Scarring Following the Faden Operation. A Retrospective Study of 100 Procedures
}

\author{
C. J. LYONS, P. FELLS, J. P. LEE, A. McINTYRE \\ London
}

\begin{abstract}
Summary
Following reports of a high incidence of chorioretinal scarring related to the Faden Procedure, a retrospective study was carried out at Moorfields Eye Hospital. One hundred unselected Faden procedures were followed up with orthoptic and fundoscopic examination. Our indications and technique for performing this operation are described. A 7\% incidence of chorioretinal scarring was noted; this compares well with previously published figures for conventional squint surgery and this operation should therefore be considered safe.
\end{abstract}

The term 'posterior fixation suture' or 'Faden operation' as it was originally known, describes the post-equatorial fixation of one of the recti to the globe by non-absorbable sutures. This results in a progressive weakening of the muscle as the globe is rotated into its field of action, without an alteration in the resting position. ${ }^{1}$

The principle of this operation was first described by Luther Peter in $1941,{ }^{2}$ who wrote of suturing a rectus muscle to the globe posterior to its insertion. The aim was to shorten the arc of contact in order to weaken the action of a muscle without a recession. The procedure as generally practised however was introduced by Cüppers ${ }^{3}$ and has been widely practised in Europe and more recently in the USA.

Some concern was therefore expressed when in February 1984 in the Archives of Ophthalmology, Alio and Faci published a prospective study of 187 eyes undergoing medial rectus Faden surgery. ${ }^{4}$ The age range of this group was 13 months to 11 years with a mean of 4.8 years \pm 2.4 . They found a $15.5 \%$ incidence of chorioretinal scarring related to the Faden sutures and concluded firstly, that this procedure is contra-indicated for the lateral rectus due to the risk of macular damage, and secondly that post-operative ophthalmoscopic examination is mandatory.

We were concerned partly because we felt this was a very high incidence of scarring and also because the conclusion of macular risk was not valid as the sutures would not be placed that far back. To our knowledge, there has been no other series in which post-Faden operation cases have been routinely examined fundoscopically but this form of surgery had always been considered safe. De Decker reported an incidence of perforation of three in 1000 operations. ${ }^{5}$ There is no indication however of whether his patients' fundi were examined post-operatively.

We undertook to assess the incidence of scarring as well as the effectiveness of the procedure in patients under the care of $\mathrm{PF}$ and JPL at Moorfields Eye Hospital.

\section{Indications for Faden surgery:}

In general terms, Faden is applied to an overacting muscle where primary alignment is 
satisfactory and incomitance increases in its direction of action.

Faden sutures may be applied:

(1) To the contralateral synergist of a paretic muscle.

(2) To the contralateral synergist in mechanical limitation (e.g. blowout fracture).

(3) To the medial recti in convergence excess esotropia which has not responded to previous treatment including bimedial recessions.

(4) To the superior rectus in DVD (alternating sursumduction) where this is a cosmetic problem.

(5) To the medial recti in nystagmus blockage syndrome.

(6) where an abnormal head posture persists following Kestenbaum surgery for nystagmus.

\section{Technique}

An experienced assistant is essential. PF always uses the operating microscope for strabismus surgery; in this procedure it provides the surgeon with a good view of the vortex veins and part of their intrascleral course.

Two bridle sutures are inserted, the conjunctiva is incised and retracted and the rectus muscle, held on a Chavasse hook, is cleaned. A Fison retractor is useful for exposure of the post-equatorial sclera and vortex veins.

A single post-equatorial bite of sclera is taken on either side of the muscle using a $1 / 2$ circle spatulate needle with $5 / 0$ Ethibond. Removal of the lid speculum is useful at this stage when operating on the superior recti. If a recession is to be combined with the Faden procedure, this is performed next. To main-

Table I Indications for Faden procedures followed up in this series

\begin{tabular}{lc}
\hline Indication & Procedures \\
\hline DVD & 27 \\
Paresis & 23 \\
Convergence excess & 16 \\
Blowout & 10 \\
Mechanical (Browns etc) & 7 \\
Nystagmus & 9 \\
Miscellaneous & 8 \\
Total & 100 \\
\hline
\end{tabular}

tain a good leverage on the globe once the muscle has been dis-inserted, a traction suture is passed through the muscle insertion. The rectus muscle is sutured to the sclera with $5 / 0$ Vicryl in its recessed position and the Ethibond sutures are passed through the lateral $1 / 5$ th of the overlying muscle. The muscle is not dis-inserted if it is not to be recessed.

\section{Present study}

All patients who underwent Faden surgery at Moorfields Eye Hospital between 1981 and 1988 were recalled at least two weeks postoperatively. Each patient underwent a full orthoptic examination following which both pupils were dilated with tropicamide $1 \%$. Fundoscopy was performed with the indirect opthalmoscope.

Any equatorial or post-equatorial chorioretinal scar in the appropriate quadrant was taken as positive. Therefore, our results would tend to over-estimate the prevalence of scarring due to the Faden operation.

\section{Characteristics of group studied}

The age at operation ranged from under one year to 50 years with a median value of 12 years.

A total of 100 procedures were followed up. In 30 of these, two bites of sclera were taken with each suture (i.e. four bites per eye). The number of procedures performed for each indication are illustrated in Table I.

The muscles operated were as follows:

$\begin{array}{ll}\text { Superior rectus } & =45 \\ \text { Medial rectus } & =34 \\ \text { Lateral rectus } & =13 \\ \text { Inferior rectus } & =8\end{array}$

Post-operative follow-up ranged from less than one year to 10 years with a median value of three years.

Approximately half the operations were performed by consultants and the rest by residents and no complications were recorded in the operation notes.

\section{Fundoscopy results}

Seven eyes were found to have chorioretinal scarring in the appropriate quadrant. The scars were densely pigmented lesions surrounded by choroidal atrophy (see Fig. 1). In none of these was there any sign of retinal 


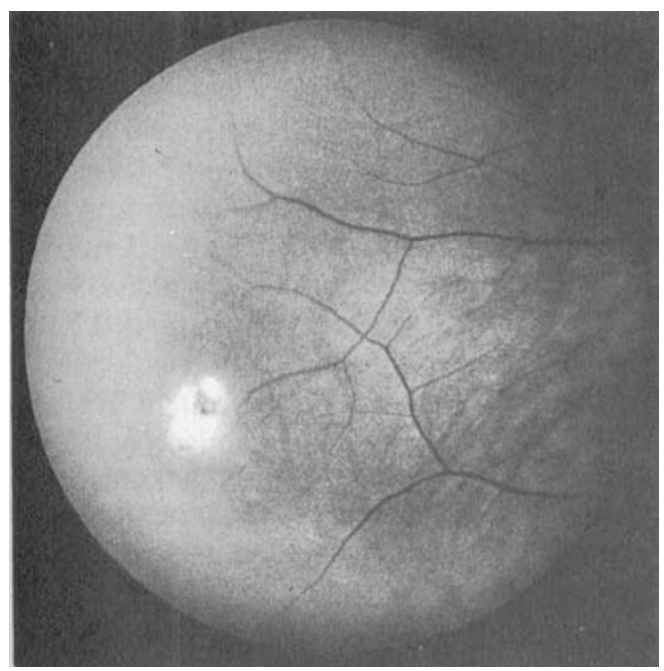

Fig.1. Chorioretinal scarring related to a Faden.

break formation or any other damage such as evidence of choroidal infarction. No damage was recorded to the vortex veins and no visual loss was recorded as a result of these lesions.

\section{Motility results}

We found the Faden procedure most useful in DVD and cases of muscle paresis. There was a marked improvement in the nystagmus group following the procedure but in mechanical limitation (Blowout, Duane, Brown's syndrome) and convergence excess esotropia, the results were poor. The disappointing results obtained in mechanical limitations, especially blowout fractures may reflect the fact that the patients considered were all under the age of 20 at the time of injury. ${ }^{6}$

\section{Conclusions}

This study suggests that the Faden operation is a safe procedure in our hands since the $7 \%$ prevalence of chorioretinal scarring compares favourably with previously published figures of $8-10 \%$ for standard recessions. ${ }^{7.8 .9}$ The sclera is at its thinnest just posterior to the rectus insertions and one would therefore expect a higher incidence of perforation when suturing in this site than at the equator where the sclera is relatively thick.

It is also interesting to note that no damage was recorded to the vortex veins despite their proximity to the suture site. The use of $5 / 0$ Ethibond with a spatulate $1 / 2$ circle needle as opposed to $4 / 0$ Mersilene, and the relatively older age group on whom we were operating may account for the discrepancy between our results and those previously published on this subject.

\section{References}

' Scott AB: the Faden operation: Mechanical effects. Am Orthop J 1977, 27: 44-7.

2 Peter LC: The extra-ocular muscles. A clinical study of normal and abnormal ocular motility. 3rd edition. London, Henry Kimpton, 1941. 324-5.

${ }^{3}$ Cüppers C: The so-called 'fadenoperation', in Fells $P$ (ed): Second Congress of the International Strabismological Association. Marseille, France. Diffusion Generale de Librairie 1974, 395-400.

${ }^{4}$ Alio $\mathrm{JL}$ and Faci A: Fundus changes following Faden Operation. Arch Opthalmol 1984, 102: 211-3.

${ }^{5}$ De Decker W: The Faden operation. When and how to do it. Trans Ophthalmol Soc UK. 1981, 101: 264.

${ }^{6}$ McCarry B, Fells P, Waddell E: Difficulties in the management of orbital blowout fractures in patients under 20 years old. In Ravault AP and Lenk M. (eds) LIPS Lyon. Transactions of the Fifth International Orthoptic Congress. 1984, 283-7.

${ }^{7}$ Gottlieb F and Castro J: Perforation of the globe during strabismus surgery. Arch Ophthalmol 1970, 84: 151-7.

${ }^{8}$ Rojas B, Vargas A, Riveros M: Periferia retiniana en operaciones de estrabismo. Arch Chil Oftalmol 1979, 36: 119-21.

${ }^{9}$ Kakuzny J, Raskewicz H, Persikiewicz A et al: Fundus periphery after operation for squint. Klin Oczna 1977, 47: 557-8. 